\title{
Cholesterol is the major component of native lipoproteins activating the p38 mitogen-activated protein kinases
}

\author{
Iveta Dobreva', Olaf Zschörnig², Gérard \\ Waeber $^{3}$, Richard W. James ${ }^{4, a}$ and Christian \\ Widmann ${ }^{1, a, *}$
}

${ }^{1}$ Département de Biologie Cellulaire et de Morphologie (DBCM), Université de Lausanne, $\mathrm{CH}-1005$ Lausanne, Switzerland

${ }^{2}$ University of Leipzig, Institute for Medical Physics and Biophysics, D-04107 Leipzig, Germany

${ }^{3}$ Département de Médecine Interne, Centre Hospitalier Universitaire Vaudois (CHUV), $\mathrm{CH}-1011$ Lausanne, Switzerland

${ }^{4}$ Lipid Laboratory, Clinical Diabetes Unit, University Hospital, CH-1211 Geneva, Switzerland

${ }^{*}$ Corresponding author

e-mail: Christian.Widmann@unil.ch

\begin{abstract}
Elevated low-density lipoprotein (LDL) levels induce activation of the p38 mitogen-activated protein kinase (MAPK), a stress-activated protein kinase potentially participating in the development of atherosclerosis. The nature of the lipoprotein components inducing p38 MAPK activation has remained unclear however. We show here that both LDLs and high-density lipoproteins (HDLs) have the ability to stimulate the p38 MAPKs with potencies that correlate with their cholesterol content. Cholesterol solubilized in methyl- $\beta$-cyclodextrin was sufficient to activate the p38 MAPK pathway. Liposomes made of phosphatidylcholine (PC) or sphingomyelin, the two main phospholipids found in lipoproteins, were unable to stimulate the p38 MAPKs. In contrast, PC liposomes loaded with cholesterol potently activated this pathway. Reducing the cholesterol content of LDL particles lowered their ability to activate the p38 MAPKs. Cell lines representative of the three main cell types found in blood vessels (endothelial cells, smooth muscle cells and fibroblasts) all activated their p38 MAPK pathway in response to LDLs or cholesterol-loaded PC liposomes. These results indicate that elevated cholesterol content in lipoproteins, as seen in hypercholesterolemia, favors the activation of the stress-activated p38 MAPK pathway in cells from the vessel wall, an event that might contribute to the development of atherosclerosis.
\end{abstract}

Keywords: cholesterol; HDL; LDL; lipoproteins; p38 MAPK; signal transduction.

\section{Introduction}

The best described physiological function of LDLs is to transport cholesterol to target tissues (Dietschy and Tur-

\footnotetext{
a The last two authors share senior authorship.
}

ley, 2002). LDLs deliver their cholesterol cargo to cells following their interaction with the LDL receptor (Dietschy and Turley, 2002). LDLs, when their vascular concentrations increase, have also been implicated in pathologies such as atherosclerosis. Elevation of LDL levels in plasma results in their retention and accumulation in atherosclerotic plaques (Glass and Witztum, 2001). The presence of LDLs in plaques apparently modulates their stability and can favor rupture of the fibrous cap and deposition of thrombus leading to acute myocardial infarction (LaBelle and Tulenko, 2004).

Besides their function as cholesterol carriers, LDLs have the potential to activate different intracellular signaling pathways such as the p38, the c-Jun N-terminal kinase (JNK) and the extracellular signal-regulated kinase (ERK) MAPKs, protein kinase C (PKC), G-proteins and cAMP (Gouni-Berthold and Sachinidis, 2002). The implication of these various cellular pathways in the physiological and pathological functions of LDLs is unclear at the present time.

The ability of LDLs to activate the p38 MAPK pathway has been observed in various cell types. For example, LDLs induce activation of the p38 MAPKs in human umbilical vein endothelial cells (HUVEC) (Zhu et al., 2001), vascular smooth muscle cells (VSMC) (Gouni-Berthold et al., 2001; Gouni-Berthold and Sachinidis, 2002), and fibroblasts (Dobreva et al., 2003). Activation of the p38 MAPK pathway was shown to be important for the upregulation of endothelial genes implicated in atherogenesis (Zhu et al., 2001), VSMC growth (Gouni-Berthold et al., 2001; Gouni-Berthold and Sachinidis, 2002), and fibroblast spreading (Dobreva et al., 2003), mechanisms that could be potentially involved in the complications of atherosclerosis. Relatively little is known however on the mechanisms that allow LDLs to activate the p38 MAPK pathway. The LDL receptor does not seem to be involved as cells lacking functional LDL receptors have the same ability as control cells to induce the p38 MAPK pathway in response to LDLs (Dobreva et al., 2003). Thus, characterization of the LDL components involved in the induction of the p38 MAPK pathway would contribute to a better understanding of the signaling properties of lipoproteins.

LDL particles are high molecular weight complexes made of esterified and non-esterified cholesterol, various phospholipids, triglycerides (TGs) and a single copy of apolipoprotein B-100 (Hevonoja et al., 2000). The presence of multiple components in LDL particles, the fact that these compounds can be oxidized and acquire new properties, and the observation that LDLs can carry trace amounts of bioactive molecules such as lysophosphatidylcholine, phosphatidylethanolamine, diacylglycerol, ceramide and some phosphoinositides (Hevonoja et al., 2000), has greatly complicated the identification of the relevant LDL components that mediate activation of the 
p38 MAPKs. Here, we present evidence that cholesterol is the main component in native lipoproteins that mediates their ability to stimulate the p38 MAPK pathway.

\section{Results}

\section{Correlation between the amount of cholesterol in lipoproteins and their ability to stimulate the p38 MAPK pathway}

To assess whether there is a shared component among various lipoprotein fractions that mediates activation of the p38 MAPK, we compared the ability of LDLs and HDLs to stimulate this pathway. As shown in Figure 1A, both lipoproteins activated the p38 MAPK pathway. Since HDLs and LDLs have a different protein composition (LDLs contain apolipoprotein B-100 and HDLs contain apolipoproteins $A, C, D, E$ and J), this indicates that the protein moiety of lipoproteins is unlikely to mediate the activation of the p38 MAPKs. In support of this notion is the observation that the two lipoproteins display different potencies in the activation of the p38 MAPK pathway when their concentration is expressed in terms of $\mu \mathrm{g} / \mathrm{ml}$ protein (Figure 1B).

If there is a shared component in the two lipoproteins that mediates the p38 response, similar dose-response curves should be obtained when their ability to activate the p38 MAPKs is expressed as a function of the concentration of the shared component. The activation of the p38 MAPKs by LDLs and HDLs determined in Figure 1B was therefore expressed as a function of the theoretical concentrations of various components present in both lipoproteins [phospholipids, cholesterol, TGs (Kritchevsky, 1986) and sphingosine-1-phosphate (S1P) (Okajima, 2002)] (Figure 1C). The data were also expressed as a function of particle concentration (Ginsberg and Goldberg, 1998) to determine whether the p38 MAPK response is proportional to the number of lipoprotein particles. Figure 1D shows that LDL and HDL had the same potency in inducing p38 MAPKs when cholesterol, but not the other components, was used to express their concentration. Moreover, when the stimulation of the p38 MAPKs by LDLs and HDLs was plotted against the actual cholesterol concentrations measured in these lipoprotein preparations, no significant difference could be detected between the lines fitting the LDL and HDL data sets (Figure 1E). Altogether these results suggest that cholesterol is a major mediator of the activation of p38 MAPKs by lipoproteins.

\section{LDL-derived lipids activate the p38 MAPKs}

If cholesterol is mediating the ability of lipoproteins to stimulate the p38 MAPK pathway, the lipid fraction extracted from LDLs, which contains unesterified and esterified cholesterol, various phospholipids, and TGs, but no proteins, should still stimulate the activation of the p38 MAPKs. Figure 2A shows that, indeed, proteindepleted LDL extracts are potent stimulators of this pathway. Moreover, pure cholesterol solubilized in meth$\mathrm{yl}-\beta$-cyclodextrin dose-dependently activated the p38 MAPKs (Figure 2B), further demonstrating the capacity of cholesterol to stimulate this MAPK pathway. In contrast, the two main phospholipids borne by lipoproteins, $\mathrm{PC}$ and sphingomyelin, did not have the capacity to activate the p38 MAPKs (Figure $2 \mathrm{C}$ and $\mathrm{D}$ ). These results indicate that it is the cholesterol moiety of lipoproteins rather than the phospholipids that is required for p38 activation.

\section{Phospholipid liposomes loaded with cholesterol strongly activate the p38 MAPKs}

In order to create a physiologically more relevant model of lipoprotein particles and study the ability of cholesterol to mediate the activation of the p38 MAPK pathway, we generated phospholipid liposomes loaded or not with cholesterol. Figure $3 \mathrm{~A}$ shows that $\mathrm{PC}$ sonicated in the presence or in the absence of cholesterol or cholesterol esters formed liposomes, while sonication of cholesterol alone did not. FACS analysis of the liposomes demonstrated a large heterogeneity in size (Figure 3B), but both small and large liposomes contained cholesterol providing that $\mathrm{PC}$ was sonicated in the presence of cholesterol or cholesterol esters (Figure $3 C$ ). Activation of the p38 MAPK pathway was induced when cells were incubated with PC liposomes containing cholesterol, but not in response to liposomes made of $\mathrm{PC}$ alone or made of $\mathrm{PC}$ and cholesterol esters (Figure 3D). These results show that the presence of unesterified cholesterol in phospholipid liposomes is essential for the activation of the p38 MAPK pathway.

\section{LDLs with lower cholesterol content display reduced ability to stimulate the p38 MAPK pathway}

To assess the importance of the cholesterol moiety in native LDL particles for the activation of the p38 MAPK pathway, we modulated their cholesterol content by incubating them in the presence of TGs. In these conditions, exchange of cholesterol for TGs occurs (Granot et al., 1985) reducing the cholesterol/protein ratio of the particles (Figure 4A, column on the right). The LDLs with lower cholesterol content had a reduced ability to stimulate the p38 MAPKs compared to native LDL particles (Figure 4A and $B$ ). This suggests that cholesterol is the limiting component in lipoprotein particles that activates the p38 MAPK pathway.

\section{Perturbation of cholesterol levels in cellular membranes stimulates the p38 MAPK pathway}

Perturbation of the lipid plasma membrane composition might be involved in activation of the p38 MAPK pathway. To experimentally modulate the cholesterol content of cell membranes, fibroblasts were incubated with the cholesterol-depleting agent methyl- $\beta$-cyclodextrin (Furuchi and Anderson, 1998; Jans et al., 2004). As shown in Figure 5, this compound induced p38 MAPK activity in a dose-dependent manner. Stimulation of the p38 MAPKs was also observed with nystatin, another cholesteroldepleting agent (data not shown). Activation of the p38 MAPK pathway upon cholesterol depletion has been reported in other cell types (e.g., keratinocytes) (Jans et al., 2004). The observations that both cholesterol-load- 
A

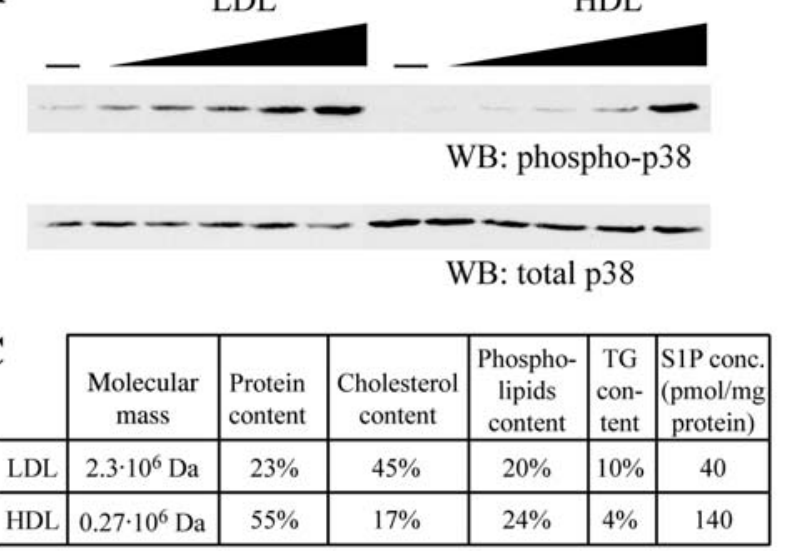

B

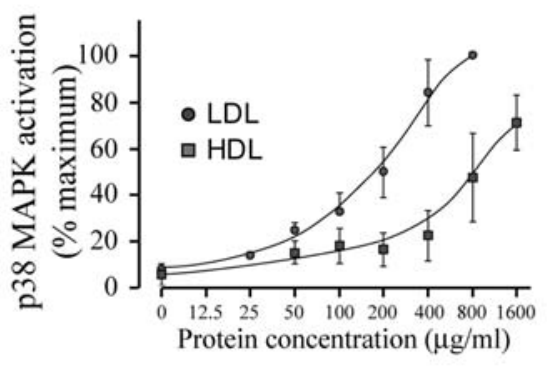

D
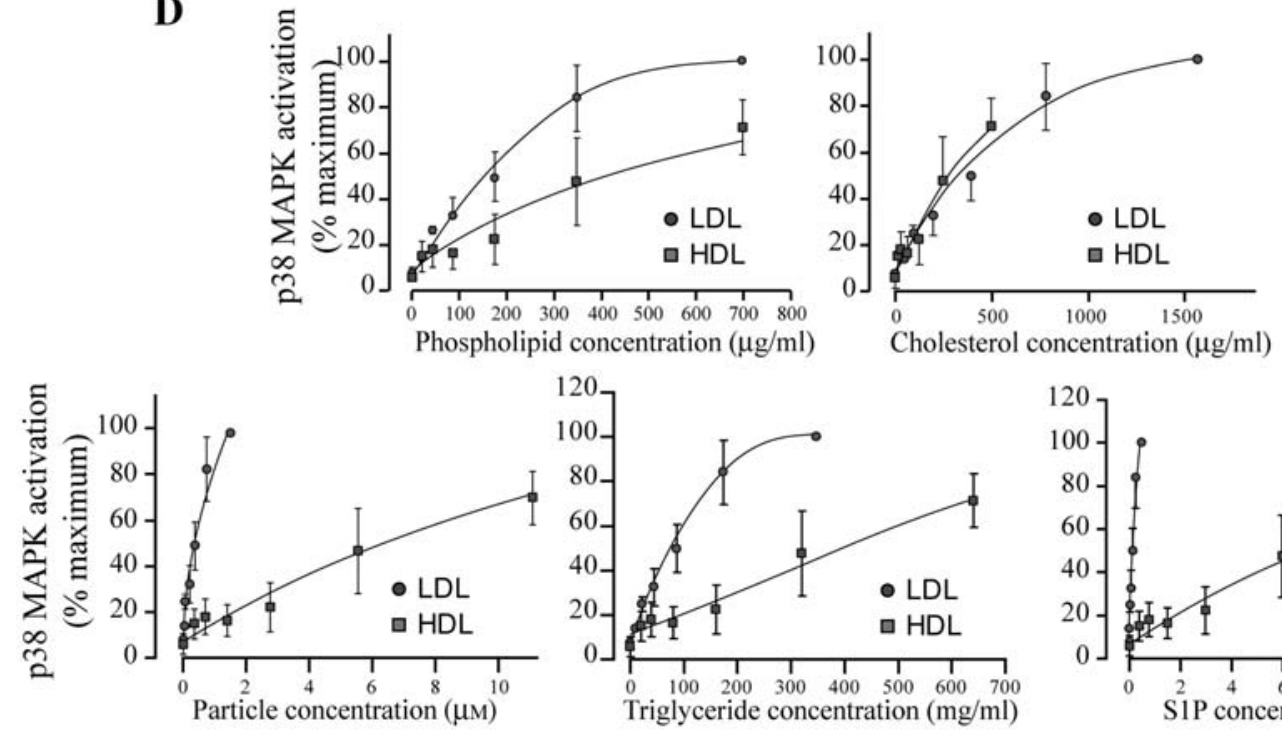

$\mathbf{E}$

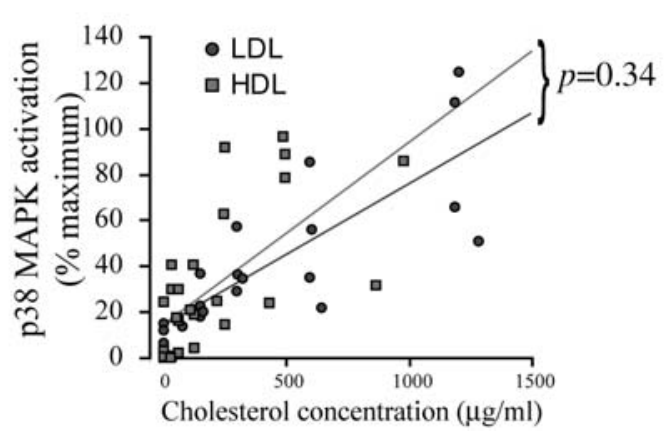

Figure 1 Correlation between the cholesterol content in lipoproteins and activation of the p38 MAPK pathway.

(A) Human fibroblasts (GM00316B) were starved for $24 \mathrm{~h}$ in lipoprotein-free medium and stimulated with increasing concentrations of LDLs $(0,50,100,200,400$, and $800 \mu \mathrm{g} / \mathrm{ml})$ or HDLs $(0,100,200,400,800$ and $1600 \mu \mathrm{g} / \mathrm{ml})$ for $10 \mathrm{~min}$. The activation of p38 MAPKs was assessed by Western blot analysis using anti-phospho-p38 MAPK antibody. Total p38 MAPK expression was also assessed by Western blot analysis.

(B) Starved human fibroblasts were stimulated with the indicated concentrations of LDLs and HDLs. The activation of the p38 MAPKs was then determined as described in panel (A). The results correspond to the quantitation of the intensity of the phospho-p38 MAPK band and are expressed as the percent of the maximum stimulation (mean \pm standard deviation of five independent experiments).

(C) Theoretical composition of the indicated components in LDL and HDL particles. See text for details.

(D) The activation of p38 obtained in Figure 1B was expressed as a function of the theoretical amounts of cholesterol, phospholipids, TGs, S1P and particle number present in LDL and HDL.

(E) The activation of p38 MAPKs induced by non-saturating concentrations of LDLs and HDLs (less than $1500 \mu \mathrm{g} / \mathrm{ml}$ ) was expressed as a function of the cholesterol concentrations measured in three independent LDL and HDL preparations (data are derived from four independent experiments and presented as described in panel B). The best-fit regression lines for the LDL and HDL data points are indicated. Linear regression analysis showed no significant difference between the slopes of these lines $(p=0.34)$. 
A

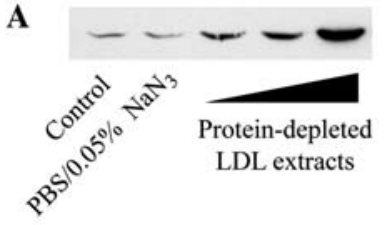

C

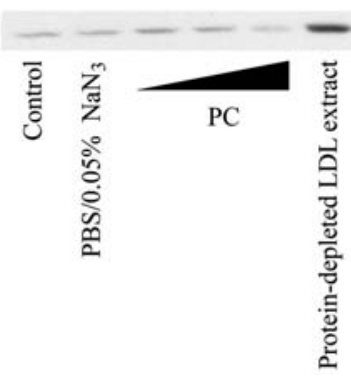

B

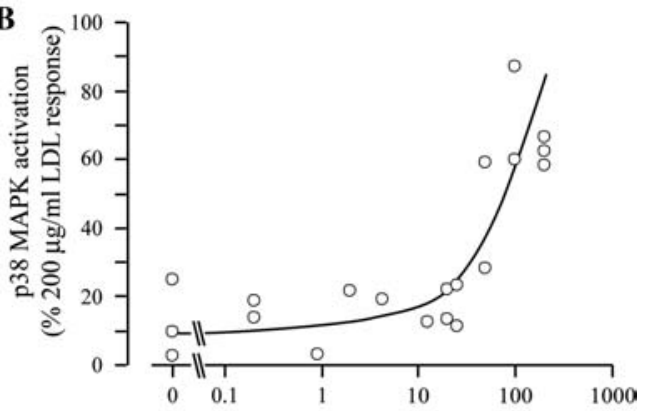

D

Cholesterol concentration $(\mu \mathrm{g} / \mathrm{ml})$

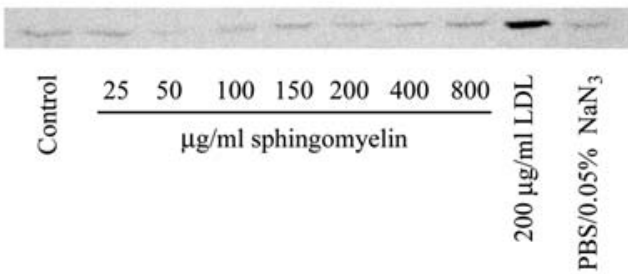

Figure 2 Cholesterol, but not PC or sphingomyelin, activates p38.

(A) Human fibroblasts were starved as in Figure $1 \mathrm{~A}$ and treated with LDL-derived lipids $-87,174$ and $348 \mu \mathrm{g} / \mathrm{ml}$ phospholipids (the corresponding amounts of phospholipids found in 100, 200 and $400 \mu \mathrm{g} / \mathrm{ml}$ LDL-protein) for $10 \mathrm{~min}$. The extent of p38 activation was assessed as in Figure 1A. This experiment has been repeated seven times with similar results.

(B) Human fibroblasts were starved as above and treated with increasing concentrations of cholesterol solubilized in $\beta$-methylcyclodextrin or $200 \mu \mathrm{g} / \mathrm{ml}$ LDL-protein for $10 \mathrm{~min}$. The extent of p38 MAPK activation was then determined. Results are normalized for p38 phosphorylation induced by the LDLs. The graph represents pooled data from three independent experiments.

(C) Human fibroblasts were starved as above and treated with increasing concentrations of PC $(0,174,393,768 \mu \mathrm{g} / \mathrm{ml})$ for $10 \mathrm{~min}$. LDL-derived lipids were used as a positive control for p38 MAPK activation. The active form of p38 MAPK was detected as above. This experiment was repeated 13 times with similar results.

(D) Human fibroblasts were starved as above and treated with increasing concentrations of sphingomyelin (SM) for 10 min. Activation of the p38 MAPKs was detected as described above. LDLs $(200 \mu \mathrm{g}$ protein/ml) were used as a positive control for p38 MAPK activation. This experiment was repeated two times with similar results.

ed vesicles and cholesterol-depleting agents stimulate the p38 MAPK pathway suggest that increasing or lowering cholesterol levels in plasma membranes induces activation of this pathway.

\section{Activation of p38 MAPKs in response to LDLs and cholesterol is detected in several blood vessel cell types}

To determine whether cell types found in blood vessels other than fibroblasts could respond to elevated cholesterol levels, a smooth muscle cell line $(A-10)$ and an endothelial cell line (HUVEC) were also analyzed. As shown in Figure 6, native LDLs (panel A) and cholesterolcontaining PC liposomes (panel B), but not empty PC liposomes (panel $B$ ), induced the activation of the p38 MAPK pathway in the three cell lines tested. These data indicate that cell lines characteristic of major cell types found in blood vessel walls (fibroblasts, smooth muscle cells and endothelial cells) efficiently induce p38 MAPKs in response to the cholesterol moiety found in lipoproteins.

\section{Contribution of other MAPK pathways to lipoprotein-induced p38 MAPK activation}

Finally, to determine if the JNK or the ERK MAPK pathways could modulate the activation of p38 MAPKs by lipoproteins, various chemical inhibitors were employed: SB 203580 that inhibits the p38 MAPKs (Davies et al.,
2000), SP 600125 that blocks all three JNK isoforms (Bennett et al., 2001), and PD 98059 that inhibits MEK1, an upstream activator of ERKs (Davies et al., 2000). Dose-response analyses were first performed to determine at which concentrations these inhibitors specifically block the relevant pathways. Serum, UV irradiation, and hyper-osmolarity $(0.5 \mathrm{M}$ sorbitol) were used to activate the ERK, JNK and p38 MAPK pathways, respectively. Figure 7A shows that $3 \mu \mathrm{M}$ SB 203580, $7 \mu \mathrm{M}$ SP 600125, and $5 \mu \mathrm{M}$ PD 98059 efficiently inhibited the pathway they were supposed to block but did not inhibit the other two MAPK pathways. These doses of inhibitors were then tested for their ability to modulate LDL-induced p38 MAPK activity. To measure the kinase activity of p38 MAPKs, phosphorylation of MAPKAP-2, a known p38 MAPK substrate, was assessed. The p38 MAPK inhibitor, but not the ERK and JNK inhibitors, abrogated the ability of p38 MAPKs to phosphorylate MAPKAP-2 in response to LDL stimulation (Figure 7B). Therefore, lipoproteininduced p38 MAPK activity does not appear to be modulated by the JNK and the ERK MAPK pathways.

SB 203580, by competitively binding to the ATP pocket of p38 MAPKs (Eyers et al., 1998), blocks their kinase activity but should not prevent their phosphorylation by upstream kinases. Figure 7B shows, however, that SB 203580 slightly reduced the ability of LDLs to induce p38 MAPK phosphorylation (the other inhibitors had no effect on LDL-stimulated p38 MAPK phosphorylation). Since p38 MAPK can undergo autophosphorylation in certain conditions, which is blocked by SB 203580 (Ge et al., 
A

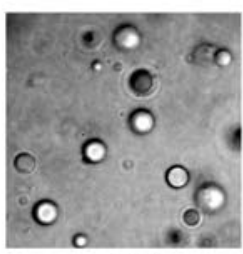

PC

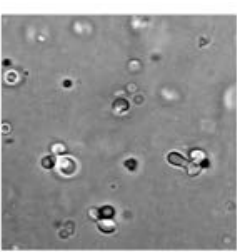

PC:cholesterol

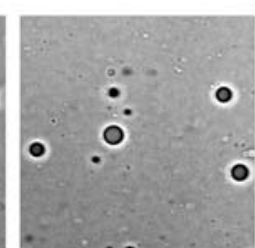

PC:esterified cholesterol

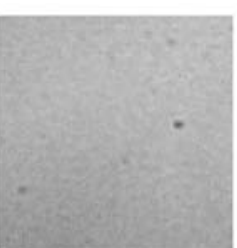

Cholesterol

B

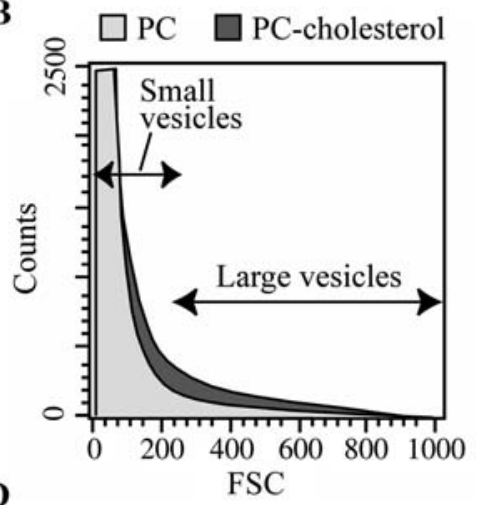

C

\begin{tabular}{|c|c|}
\hline Vesicle type & $\begin{array}{c}\text { Cholesterol content in } \\
10 \cdot 10^{6} \text { purified vesicles }\end{array}$ \\
\hline PC & $0 \mu \mathrm{g}$ \\
\hline PC:cholesterol & $\begin{array}{c}\text { Small vesicles: } 12 \mu \mathrm{g} \\
\text { Large vesicles: } 81 \mu \mathrm{g}\end{array}$ \\
\hline $\begin{array}{c}\text { PC: } \text { esterified } \\
\text { cholesterol }\end{array}$ & $\begin{array}{c}\text { Small vesicles: } 2 \mu \mathrm{g} \\
\text { Large vesicles: } 11 \mu \mathrm{g}\end{array}$ \\
\hline Cholesterol & $\begin{array}{c}\text { Not relevant } \\
\text { (no vesicle formed) }\end{array}$ \\
\hline
\end{tabular}

D

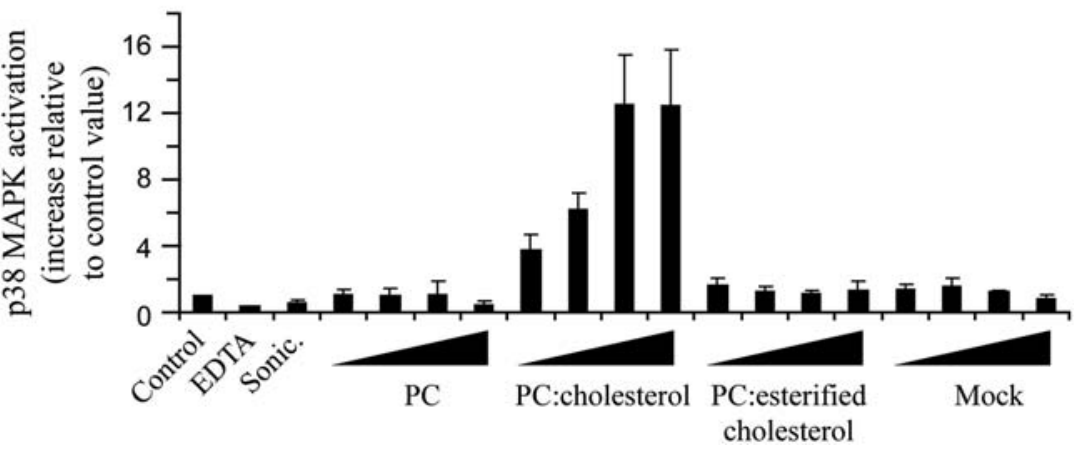

Figure 3 PC liposomes containing unesterified cholesterol are potent activators of the p38 MAPKs.

(A) Phase contrast images of the indicated sonicated mixtures.

(B) Liposomes made of PC alone or PC and either cholesterol or cholesterol esters were submitted to FACS analysis (light gray profile: PC only liposomes; dark gray profile: PC-cholesterol liposomes).

(C) 10 million of the small and large liposome vesicles indicated in panel (B) were purified using a cell sorter and their cholesterol content measured.

(D) Human fibroblasts were starved as described in Figure 1A and treated for 10 min with PBS (control), PBS/0.1 mM EDTA (EDTA), sonicated PBS/0.1 mM EDTA (sonic.), increasing concentrations of empty PC liposomes or PC liposomes containing cholesterol or cholesterol esters. The amounts of liposomes used correspond to the PC content in the liposomes (100, 200, $400 \mathrm{and} 800 \mu \mathrm{g} / \mathrm{ml}$ PC or the corresponding volume of the supernatant of a cholesterol suspension in PBS [mock]). The cells were then lysed and activation of the p38 MAPKs was assessed by Western blot analysis using anti-phospho-p38 MAPK antibody. The results are expressed as fold increase relative to the control value (mean $\pm S D$ of three independent experiments).

2002), it is possible that lipoproteins induce p38 MAPK activity not only by stimulating the conventional p38 MAPK kinases (i.e., MKK3 and MKK6) but also by favoring autophosphorylation of p38 MAPKs.

\section{Discussion}

Lipoproteins, which are the main cholesterol suppliers for cells, are known to affect MAPK pathways in various cell types (Gouni-Berthold and Sachinidis, 2002). However, the nature of the components within the lipoprotein particles that mediate activation of the MAPK pathways has not been clearly established. We have previously shown that LDLs activate the p38 MAPK pathway in fibroblasts in an LDL receptor-independent manner (Dobreva et al.,
2003). These results indicate that there are other receptors for LDL particles that mediate their ability to activate the p38 MAPKs or that the lipoproteins do not need to be internalized to activate these MAPKs. In the latter case, transfer of lipoprotein components to the plasma membrane could be the biochemical event leading to activation of the p38 MAPKs.

While some components of lipoproteins, including PC and sphingomyelin, do not activate the p38 MAPK pathway (Figure 2), other components of lipoproteins, or molecules carried by lipoproteins, have the potential to stimulate this pathway. For example, some phospholipid derivates, such as lysophosphatidylcholine (LPC) and lysophosphatidic acid (LPA), can activate the p38 MAPK pathway (Jing et al., 2000; Hayashi et al., 2001; Takahashi et al., 2002). However, these components are either 


\begin{tabular}{lccc} 
A & & LDL-protein & $\begin{array}{c}\text { Cholesterol/ } \\
\text { Protein ratio }\end{array}$ \\
\hline Prep \#1 & native & 2.6 \\
& treated & 1.8 \\
\hline \multirow{2}{*}{ Prep \#2 } & native & treated & 2.7 \\
\hline & native & \\
\hline & treated & \\
\hline
\end{tabular}

\section{B}

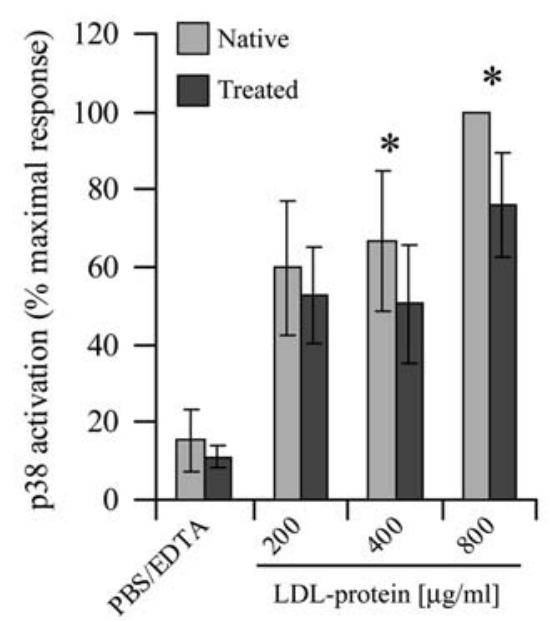

Figure 4 LDLs with lower cholesterol content display decreased ability to activate the p38 MAPK pathway.

(A) Human fibroblasts were starved as described in Figure $1 \mathrm{~A}$ and treated with increasing concentrations of native LDLs or LDLs with lower cholesterol content (see materials and methods). Activation of the p38 MAPKs was measured as described in Figure 1A. Shown are representative blots from three different sets of native LDL and LDLs with lower cholesterol content. The cholesterol/protein ratios for each set are shown on the right. (B) Quantitation of the data presented in panel (A). Results correspond to the mean $\pm S D$ of four independent experiments.

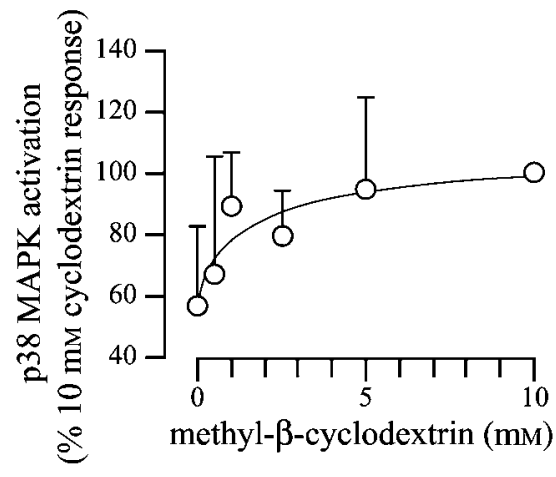

Figure 5 Depletion of cholesterol in plasma membranes induces p38 MAPK activation.

Human fibroblasts were starved as above and treated with the indicated concentrations of methyl- $\beta$-cyclodextrin for $10 \mathrm{~min}$. Activation of the p38 MAPKs was detected as described above. Results are normalized to the value obtained with $10 \mathrm{~mm}$ cyclodextrin and correspond to the average of four independent experiments. found in small amounts or not present at all in native LDL particles. Since we did not detect oxidation in our LDL preparations (data not shown; Dobreva et al., 2003) and since oxidation of LDLs is not required for the activation of the p38 MAPKs (Dobreva et al., 2003), it is unlikely that LPC, LPA or other oxidized derivates of LDL components play a major role in the modulation of the p38 MAPK pathway by native lipoproteins.

$\mathrm{S} 1 \mathrm{P}$ is yet another phospholipid that has the potential to mediate the activation of p38 MAPKs (Robert et al., 2001). There is however no correlation between the ability of LDLs and HDLs to induce p38 MAPK activation when their concentrations are expressed in terms of their S1P content (Figure 1). In addition, the amount of S1P necessary for the activation of the p38 MAPKs is well above the concentrations found in native lipoproteins (Robert et al., 2001). This suggests that although some phospholipid derivates have the ability to activate the p38 MAPKs at pharmacological concentrations they are unlikely to be the main mediators of lipoprotein-induced p38 MAPK activation.

The present study indicates that cholesterol is the main lipoprotein component responsible for the activation of the p38 MAPK pathway in three major cell types found in blood vessels, i.e., fibroblasts, smooth muscle cells and endothelial cells. Several observations support this notion: (i) LDL and HDL have the same potency in inducing p38 MAPKs when cholesterol, but not other components, is used to express their concentration; (ii) cholesterol solubilized in methyl- $\beta$-cyclodextrin or incorporated in phospholipid liposomes stimulates the p38 MAPKs, and (iii) reduction in the cholesterol content in LDL particles diminishes their ability to stimulate the p38 MAPK pathway.

The mechanism used by lipoprotein cholesterol to stimulate the p38 MAPKs is unclear at the present time. LDL receptor family members do not seem to be involved as lipoproteins efficiently stimulate the p38 MAPKs in conditions where this receptor family is absent or inhibited (Dobreva et al., 2003). In fact, lipoproteins may not need to be internalized to deliver cholesterol to cell membranes. For example, the scavenger receptor type $\mathrm{BI}$ (SR-BI), while binding to lipoproteins, does not internalize most of them (Fidge, 1999). However it does allow the transfer of cholesterol from the particles to cellular membranes (Fidge, 1999; Cherradi et al., 2001; Eckhardt et al., 2004). This can lead to changes of the bio-physical properties of these membranes (Brown and London, 2000) and modulation of various cellular and signaling responses (Pike and Miller, 1998; Xavier et al., 1998; Sheets et al., 1999; Incardona and Eaton, 2000). It is known that cholesterol can affect receptor affinities and activities of proteins with sterol-sensing domains (Incardona and Eaton, 2000). Moreover, caveolaes and lipid rafts, corresponding to membrane domains with increased cholesterol content, are enriched in specific signaling proteins and modulate several signaling pathways including the ERK MAPK (Furuchi and Anderson, 1998) and tumor necrosis factor (TNF)-induced nuclear factor-kappa B (NF-кB) activation (Legler et al., 2003).

It will be important in the future to characterize the mechanisms by which cholesterol in lipoproteins activate 


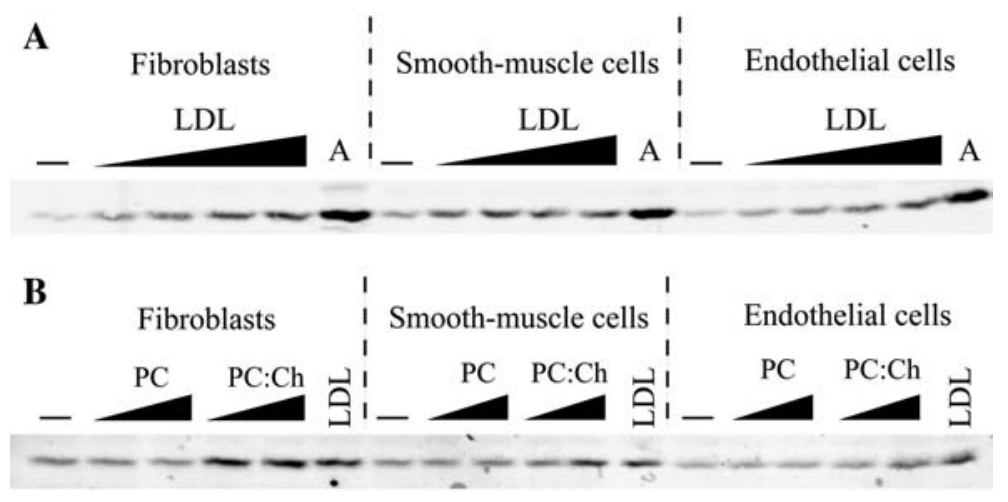

Figure 6 LDLs and cholesterol-containing liposomes activate the p38 MAPKs in fibroblasts, smooth muscle cells and endothelial cells.

(A) GM00316, A-10 and HUVEC were starved as described in Figure 1A and treated with increasing concentrations of LDLs $(0,50$, $100,200,400 \mu \mathrm{g} / \mathrm{ml}$ ) or $0.1 \mu \mathrm{g} / \mathrm{ml}$ anisomycin (A; positive control for p38 MAPK activation) for $10 \mathrm{~min}$. The cells were then lysed and the activation of the p38 MAPKs was assessed by Western blotting using anti-phospho-p38 MAPK antibodies.

(B) GM00316, A-10 and HUVEC were starved as above and treated with increasing concentrations $(0,400$, and $800 \mu \mathrm{g} \mathrm{PC} / \mathrm{ml})$ of empty PC liposomes (PC) or PC liposomes containing cholesterol (PC:Ch) or $200 \mu \mathrm{g} / \mathrm{ml}$ LDL for $10 \mathrm{~min}$. Activation of the p38 MAPKs was measured as above.

the p38 MAPK pathway and determine the contribution of this signaling event in the development of pathological conditions, such as atherosclerosis, that arise from increased lipoprotein blood levels.

\section{Materials and methods}

\section{Materials}

PC, sphingomyelin, cholesterol, cholesterol linoleate and methyl$\beta$-cyclodextrin were obtained from Sigma (Buchs, Switzerland). Alternatively, PC and cholesterol were obtained from Avanti Polar Lipids (Alabaster, AL, USA). Anti-phospho-p38 (Thr180/ Tyr182), anti-p38 MAPK, anti-phospho-cJun (Ser73), anti-phospho-ERK (Thr202/Tyr204) and anti-phospho-MAPKAP-2 (Thr334) antibodies were purchased from Cell Signaling Technology (Beverly, MA, USA). SB 203580, SP 600125 and PD 98059, were from Alexis Corporation (Lausen, Switzerland). They were dissolved in DMSO as $10 \mathrm{~mm}, 20 \mathrm{~mm}$ and $50 \mathrm{~mm}$ stock aliquots, respectively, and stored at $-20^{\circ} \mathrm{C}$. The anti-RasGAP antibody was also obtained from Alexis Corporation.

\section{Cell culture}

A-10 (rat smooth-muscle cells) and HUVEC were obtained from ATCC (via LGC Promochem, Molsheim, France). GM00316B human skin fibroblasts were purchased from Coriell Cell Repositories (Camden, NJ, USA). A-10 and GM00316 were maintained in Dulbecco's modified Eagle's medium (DMEM), supplemented with 15\% non-decomplemented fetal calf serum (FCS; Amimed, Bioconcept, Allschwil, Switzerland) at $37^{\circ} \mathrm{C}$ and $5 \% \quad \mathrm{CO}_{2}$. HUVEC were cultured in F-12K medium (Kaighn's modification of Ham's F-12, ATCC), containing $33.3 \mu \mathrm{g} / \mathrm{ml}$ endothelial cell growth supplement (Sigma), $0.1 \mathrm{mg} / \mathrm{ml}$ heparin and $10 \%$ nondecomplemented FCS.

\section{Lipoprotein and lipoprotein-free serum preparation and purification}

LDL and HDL were isolated from human serum by sequential density ultracentrifugation as described previously (Havel et al., 1955; James and Pometta, 1990) and dialyzed for $48 \mathrm{~h}$ against PBS containing $100 \mu \mathrm{M}$ EDTA. Human lipoprotein-free serum was prepared by removal of lipoproteins by ultracentrifugation at a density of $1.23 \mathrm{~g} / \mathrm{l}$ (James and Pometta, 1990). The protein concentration was measured by Bradford assay using BSA as a standard. The cholesterol concentration was measured by enzymatic in vitro test kit from Roche (Basel, Switzerland).

\section{Preparation of lipid extracts}

Total lipid extracts from LDL and HDL fractions were prepared by chloroform:methanol (2:1) extraction of lyophilized lipoproteins (Olofsson et al., 1980) for $30 \mathrm{~min}$ at $-20^{\circ} \mathrm{C}$. The solvent was evaporated under nitrogen stream and the dried lipids were resuspended by vortexing in PBS, $0.05 \% \mathrm{NaN}_{3}$.

\section{Preparation and characterization of phospholipid liposomes loaded or not with cholesterol}

PC, sphingomyelin, cholesterol and cholesterol linoleate were dissolved in chloroform. For the preparation of liposomes in Figure 3, aliquots of the phospholipid and cholesterol stock solutions [at a PC:cholesterol ratio of 1:2.5 (w/w) when these two compounds were mixed] were dried under nitrogen stream and resuspended in PBS, $100 \mu \mathrm{M}$ EDTA (or $10 \mathrm{~mm}$ HEPES pH 7.4, $100 \mathrm{~mm} \mathrm{NaCl}$ ) at $55^{\circ} \mathrm{C}$. The samples were continuously vortexed and the tubes were filled with nitrogen to avoid oxidation. The resulting suspensions were sonicated in a sonication bath for $6 \mathrm{~h}$ at $55^{\circ} \mathrm{C}$. The presence of phospholipid liposomes was confirmed by taking pictures of the liposomes with a Zeiss Axioplan 2 microscope. The liposomes were purified by FACS.

In Figure 6, the phospholipid/cholesterol suspensions were sonicated with a Branson tip sonifier for 10 min under temperature control $\left(\max .30^{\circ} \mathrm{C}\right)$. The resulting clear opalescent preparations were checked by photon correlation spectroscopy (Malvern Zetasizer 4) for size distribution. Applying a multimodal size distribution, the size heterogeneity was measured.

The amount of phosphate was measured as described (Chen et al., 1956). Cholesterol incorporation was determined as mentioned above. MALDI-TOF MS analysis of the sonicated preparations did not indicate an additional formation of lysolipids and other impurities of the samples.

\section{Modification of the cholesterol content in LDL}

LDLs were incubated with a preparation enriched in TGs for $6-8 \mathrm{~h}$ at room temperature with constant slow mixing. LDLs 
A $\quad$ SB 203580 SP 600125 PD 98059

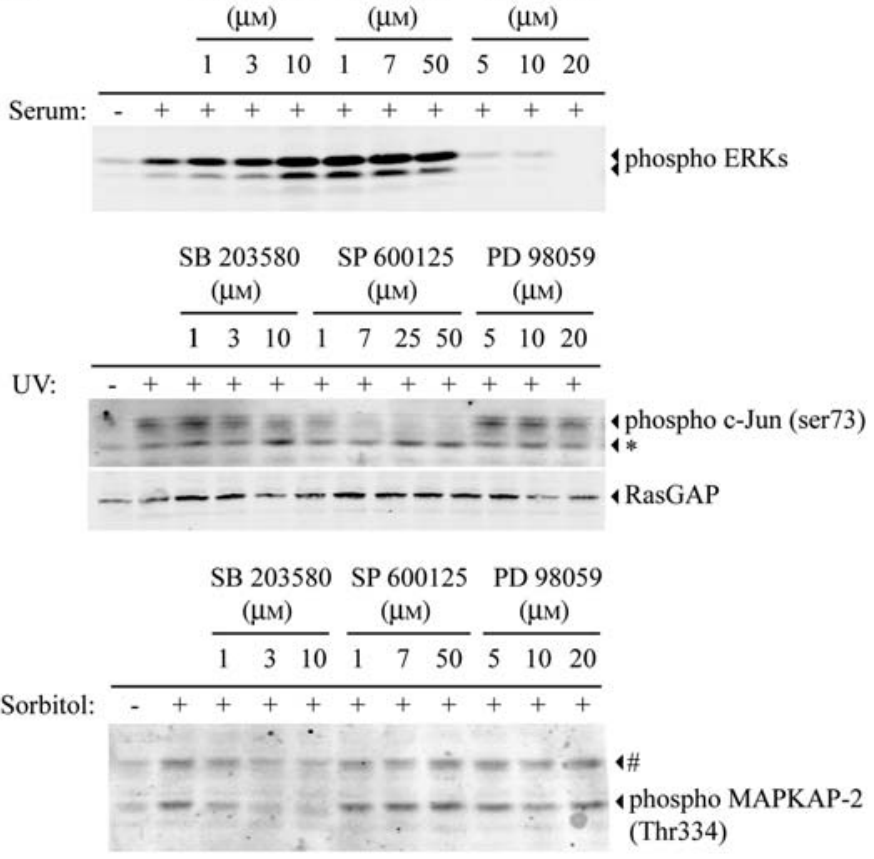

B

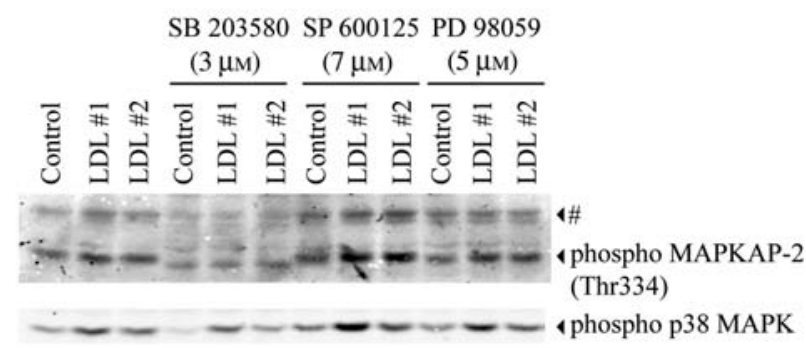

Figure 7 LDL-induced MAPKAP-2 phosphorylation is blocked by a specific p38 MAPK inhibitor.

Human fibroblasts were plated in six-well plates $\left(60 \times 10^{3}\right.$ cells/well) and the following day starved in serum-free DMEM for an additional 24-h period. The cells were preincubated with the indicated concentration of SB 203580, SP 600125 and PD 98059 for $30 \mathrm{~min}$ and stimulated with serum ( $10 \%$ for $10 \mathrm{~min})$, UV (60 J/m² followed by a 1 -h incubation) and sorbitol $(0.5 \mathrm{M} \mathrm{for} 30 \mathrm{~min})$. The cells were then lysed and phosphorylation of ERKs, c-Jun, and MAPKAP-2 assessed by Western blotting. For c-Jun detection, the cells were directly lysed in sample buffer, but this prevented accurate protein concentration measurement. A loading control (RasGAP) is therefore also presented in this case (panel A). Alternatively, the cells, following the preincubation with the inhibitors, were stimulated with $400 \mu \mathrm{g} / \mathrm{ml}$ LDL for $15 \mathrm{~min}$ (panel B). The active forms of MAPKAP-2 and p38 MAPKs were detected by Western blot analysis using anti-phospho MAPKAP-2 (Thr 334) and anti-phospho-p38 MAPK antibodies. Shown are representative blots from five independent experiments. The asterisk denotes a band that might correspond to phospho-JunD and the \# symbol indicates a band that could correspond to phospho-MAPKAP-3.

were re-isolated by centrifugation. This leads to an exchange of TGs for cholesterol resulting in lipoproteins with lower cholesterol content (Granot et al., 1985).

\section{Western blot analysis}

Cells were lysed in mono Q-C buffer (Yang and Widmann, 2001), supplemented with 1 tablet of complete EDTA free protease inhibitor per $50 \mathrm{ml}$ (Roche). For the detection of phospho-cJun, cells were directly lysed in sample buffer. Briefly, $60 \times 10^{3}$ cells were plated in six-well plates, grown for 1 day and starved in DMEM for an additional day. The cells were then subjected to the treatments described in the figures, then washed once with cold PBS and lysed in $70 \mu \mathrm{l}$ of sample buffer $(62.4 \mathrm{~mm}$ Tris- $\mathrm{HCl}$ $\mathrm{pH} 6.8,10 \%$ glycerol, $5 \% \beta$-mercaptoethanol, $2 \%$ sodium dodecyl sulfate, $0.01 \%$ bromophenol blue) on ice before being loaded on SDS-PAGE.
Protein concentration was measured by Bradford assay using BSA as a standard. Western blotting was performed as described previously (Widmann et al., 1995) using a home-made ECL reagent (Yang and Widmann, 2001). When quantitations were performed, the primary antibody was revealed with a 1/ 2500 dilution of an Alexa Fluor 680-conjugated anti-rabbit antibody (Molecular Probes, Eugene, OR, USA) or an IRDye 800-conjugated anti-mouse antibody (Rockland, Gilbertsville, PA, USA) and subsequently visualized with the Odyssey infrared imaging system (Licor, Homburg, Germany). Quantitation of the bands was performed using the Odyssey infrared imaging software.

\section{Statistics}

The statistics used in Figure 1 were performed with the SAS System for Windows (SAS Institute Inc., Cary, NC, USA) using 
linear regression analysis and those used in Figure 4 were performed with Microsoft Excel (XP edition) using the Student's $t$ test.

\section{Acknowledgments}

This work was supported by the Botnar Foundation (Lausanne, Switzerland) and grants from the Leenaards Foundation to C.W. and R.W.J. We thank Rudolph Kraftsik for his help with the statistical analyses. We also thank Estelle Devêvre and Pedro Romero (Centre coordonné d'oncologie ambulatoire, CHUV, Lausanne, Switzerland) for their assistance with the FACS.

\section{References}

Bennett, B.L., Sasaki, D.T., Murray, B.W., O'Leary, E.C., Sakata, S.T., Xu, W., Leisten, J.C., Motiwala, A., Pierce, S., Satoh, Y. et al. (2001). SP600125, an anthrapyrazolone inhibitor of Jun $\mathrm{N}$-terminal kinase. Proc. Natl. Acad. Sci. USA 98, 1368113686.

Brown, D.A. and London, E. (2000). Structure and function of sphingolipid- and cholesterol-rich membrane rafts. J. Biol. Chem. 275, 17221-17224.

Chen Jr., P.S., Toribara, T.Y., and Warner, H. (1956). Microdetermination of phosphorus. Anal. Chem. 28, 1756-1758.

Cherradi, N., Bideau, M., Arnaudeau, S., Demaurex, N., James, R.W., Azhar, S., and Capponi, A.M. (2001). Angiotensin II promotes selective uptake of high density lipoprotein cholesterol esters in bovine adrenal glomerulosa and human adrenocortical carcinoma cells through induction of scavenger receptor class B type I. Endocrinology 142, 4540-4549.

Davies, S.P., Reddy, H., Caivano, M., and Cohen, P. (2000). Specificity and mechanism of action of some commonly used protein kinase inhibitors. Biochem. J. 351, 95-105.

Dietschy, J.M. and Turley, S.D. (2002). Control of cholesterol turnover in the mouse. J. Biol. Chem. 277, 3801-3804.

Dobreva, I., Waeber, G., Mooser, V., James, R.W., and Widmann, C. (2003). LDLs induce fibroblast spreading independently of the LDL receptor via activation of the p38 MAPK pathway. J. Lipid Res. 44, 2382-2390.

Eckhardt, E.R., Cai, L., Sun, B., Webb, N.R., and Van Der Westhuyzen, D.R. (2004). High density lipoprotein uptake by scavenger receptor SR-BII. J. Biol. Chem. 279, 1437214381.

Eyers, P.A., Craxton, M., Morrice, N., Cohen, P., and Goedert, M. (1998). Conversion of SB 203580-insensitive MAP kinase family members to drug-sensitive forms by a single aminoacid substitution. Chem. Biol. 5, 321-328.

Fidge, N.H. (1999). High density lipoprotein receptors, binding proteins, and ligands. J. Lipid Res. 40, 187-201.

Furuchi, T. and Anderson, R.G. (1998). Cholesterol depletion of caveolae causes hyperactivation of extracellular signal-related kinase (ERK). J. Biol. Chem. 273, 21099-21104.

Ge, B., Gram, H., Di Padova, F., Huang, B., New, L., Ulevitch, R.J., Luo, Y., and Han, J. (2002). MAPKK-independent activation of p38a mediated by TAB1-dependent autophosphorylation of p38a. Science 295, 1291-1294.

Ginsberg, H.N. and Goldberg, I.J. (1998). Disorders of lipoprotein metabolism. In: Harrison's Principles of Internal Medicine (Berkshire, UK: McGraw Hill).

Glass, C.K. and Witztum, J.L. (2001). Atherosclerosis. The road ahead. Cell 104, 503-516.

Gouni-Berthold, I., Berthold, H.K., Weber, A.A., Seul, C., Vetter, H., and Sachinidis, A. (2001). Troglitazone and rosiglitazone inhibit the low density lipoprotein-induced vascular smooth muscle cell growth. Exp. Clin. Endocrinol. Diabetes 109, 203-209.
Gouni-Berthold, I. and Sachinidis, A. (2002). Does the coronary risk factor low density lipoprotein alter growth and signaling in vascular smooth muscle cells? FASEB J. 16, 1477-1487.

Granot, E., Deckelbaum, R.J., Eisenberg, S., Oschry, Y., and Bengtsson-Olivecrona, G. (1985). Core modification of human low-density lipoprotein by artificial triacylglycerol emulsion. Biochim. Biophys. Acta 833, 308-315.

Havel, R.J., Eder, H.A., and Bragton, J.H. (1955). The distribution and chemical composition of ultracentrifugally separated lipoproteins in human serum. J. Clin. Invest. 34, 1345-1353.

Hayashi, K., Takahashi, M., Nishida, W., Yoshida, K., Ohkawa, Y., Kitabatake, A., Aoki, J., Arai, H., and Sobue, K. (2001). Phenotypic modulation of vascular smooth muscle cells induced by unsaturated lysophosphatidic acids. Circ. Res. 89, 251-258.

Hevonoja, T., Pentikainen, M.O., Hyvonen, M.T., Kovanen, P.T., and Ala-Korpela, M. (2000). Structure of low density lipoprotein (LDL) particles: basis for understanding molecular changes in modified LDL. Biochim. Biophys. Acta 1488, 189-210.

Incardona, J.P. and Eaton, S. (2000). Cholesterol in signal transduction. Curr. Opin. Cell Biol. 12, 193-203.

James, R.W. and Pometta, D. (1990). Immunofractionation of high density lipoprotein subclasses 2 and 3 . Similarities and differences of fractions isolated from male and female populations. Atherosclerosis 83, 35-45.

Jans, R., Atanasova, G., Jadot, M., and Poumay, Y. (2004). Cholesterol depletion upregulates involucrin expression in epidermal keratinocytes through activation of p38. J. Invest. Dermatol. 123, 564-573.

Jing, Q., Xin, S.M., Zhang, W.B., Wang, P., Qin, Y.W., and Pei, G. (2000). Lysophosphatidylcholine activates p38 and p42/ 44 mitogen-activated protein kinases in monocytic THP-1 cells, but only p38 activation is involved in its stimulated chemotaxis. Circ. Res. 87, 52-59.

Kritchevsky, D. (1986). Atherosclerosis and nutrition. Nutr. Int. 2, 290-297.

LaBelle, E.F. and Tulenko, T.N. (2004). LDL, IGF-1, and VSMC apoptosis: linking atherogenesis to plaque rupture in vulnerable lesions. Cardiovasc. Res. 61, 204-205.

Legler, D.F., Micheau, O., Doucey, M.A., Tschopp, J., and Bron, C. (2003). Recruitment of TNF receptor 1 to lipid rafts is essential for TNF $\alpha$-mediated NF-кB activation. Immunity 18 , 655-664.

Okajima, F. (2002). Plasma lipoproteins behave as carriers of extracellular sphingosine 1-phosphate: is this an atherogenic mediator or an anti-atherogenic mediator? Biochim. Biophys. Acta 1582, 132-137.

Olofsson, S.O., Bostrom, K., Svanberg, U., and Bondjers, G. (1980). Isolation and partial characterization of a polypeptide belonging to apolipoprotein $B$ from low-density lipoproteins of human plasma. Biochemistry 19, 1059-1064.

Pike, L.J. and Miller, J.M. (1998). Cholesterol depletion delocalizes phosphatidylinositol bisphosphate and inhibits hormone-stimulated phosphatidylinositol turnover. J. Biol. Chem. 273, 22298-22304.

Robert, P., Tsui, P., Laville, M.P., Livi, G.P., Sarau, H.M., Bril, A., and Berrebi-Bertrand, I. (2001). EDG1 receptor stimulation leads to cardiac hypertrophy in rat neonatal myocytes. J. Mol. Cell Cardiol. 33, 1589-1606.

Sheets, E.D., Holowka, D., and Baird, B. (1999). Critical role for cholesterol in Lyn-mediated tyrosine phosphorylation of FcepsilonRI and their association with detergent-resistant membranes. J. Cell Biol. 145, 877-887.

Takahashi, M., Okazaki, H., Ogata, Y., Takeuchi, K., Ikeda, U., and Shimada, K. (2002). Lysophosphatidylcholine induces apoptosis in human endothelial cells through a p38-mitogenactivated protein kinase-dependent mechanism. Atherosclerosis $161,387-394$.

Widmann, C., Dolci, W., and Thorens, B. (1995). Agonist-induced internalization and recycling of the glucagon-like peptide-1 
receptor in transfected fibroblasts and in insulinomas. Biochem. J. 310, 203-214.

Xavier, R., Brennan, T., Li, Q., McCormack, C., and Seed, B. (1998). Membrane compartmentation is required for efficient T cell activation. Immunity $8,723-732$.

Yang, J.-Y. and Widmann, C. (2001). Antiapoptotic signaling generated by caspase-induced cleavage of RasGAP. Mol. Cell. Biol. 21, 5346-5358.
Zhu, Y., Liao, H., Wang, N., Ma, K.S., Verna, L.K., Shyy, J.Y., Chien, S., and Stemerman, M.B. (2001). LDL-activated p38 in endothelial cells is mediated by Ras. Arterioscler. Thromb. Vasc. Biol. 21, 1159-1164.

Received February 3, 2005; accepted June 9, 2005 